\title{
Where Is the Community Dimension in the Updated Common Rule?
}

\author{
Raquel Reyes, MD, MPA ${ }^{1,2,4,5}$, Carol E. Lorenz, $\mathrm{PhD}^{6}$, Stuart Rennie, $\mathrm{PhD}^{1,3,7}$, Alan Richmond, MSW', and Giselle Corbie-Smith, MD, MSc ${ }^{1,2,3,4}$ \\ (1) School of Medicine, University of North Carolina at Chapel Hill; (2) Department of Medicine, University of North Carolina at Chapel Hill; (3) Department of Social \\ Medicine, University of North Carolina at Chapel Hill; (4) Center for Health Equity Research, University of North Carolina at Chapel Hill; (5) Institute for Global Health \& \\ Infectious Disease, University of North Carolina at Chapel Hill; (6) Center for Women's Health Research, University of North Carolina at Chapel Hill; (7) Bioethics Center \\ University of North Carolina at Chapel Hill; (8) Community-Campus Partnerships for Health
}

Submitted 28 November 2016, revised 17 July 2017, accepted 17 August 2017.

\section{Abstract}

The Problem: Changes to the Federal Policy for the Protection of Human Subjects (the Common Rule) as presented in the Notice of Proposed Rulemaking (NPRM) are both logical and necessary. However, the proposed changes continue to focus entirely on the individual and fail to take into account the rapidly-emerging types of research that involve patients and communities directly in the research process.

Purpose of Article: We propose several changes and amendments that address the interests of communities and underscore the principle of justice, especially social justice.

Key Points: Our recommendations seek to revise human subjects' protections that currently overemphasize individualism

I n the 20 years since the Federal Policy for the Protection of Human Subjects (also known as the "Common Rule") was last updated, the field of research has evolved considerably. Research has become more collaborative, crossing scientific and discipline boundaries, and including groups who previously were not included owing to presumed vulnerability. Scientific knowledge on multiple fronts is proliferating rapidly. Data acquisition, collection, ownership, and the sharing and protection of data are both enhanced and complicated by advances in technology. New ways to plan and operationalize studies and analyze results are developing at a rapid rate.

In recognition of these changes in the research landscape, the Common Rule was updated and published in the Federal Register in January 2017 after public comment periods in 2011 (the Advance Notice of Proposed Rule Making) and 2015 (the Notice of Proposed Rule Making). The intent of and autonomy to reflect a collectivist ethos that would extend protections to communities engaged in medical research.

Conclusion: We believe this is necessary to effectively and efficiently conduct the types of research that will ultimately rectify health inequities that continue to exist in many communities, but particularly communities of color.

\section{Keywords}

Community-based participatory research, community health partnerships, vulnerable populations, health disparities, health outcomes

the proposed revisions was to "better protect human subjects involved in research, while facilitating valuable research and reducing burden, delay, and ambiguity for investigators." As adopted, the most significant changes to the rule include new requirements for informed consent, an option to obtain "broad consent" for future studies using stored biospecimens, new categories of exempt research, the elimination of continuing review for many low-risk studies, and a general requirement to use a single institutional review board (IRB) for multi-institution studies.

Early guidance for the Common Rule focused almost exclusively on consent and the fair distribution of burdens and benefits, and these most recent changes in the Final Rule continue in the same vein. Largely absent from both the public discourse and the updated guidelines is a recognition of the importance of community-level considerations in research. The 
guidelines and requirements established in the Common Rule are set on centuries of a sordid past in research, where science has been misused to harm and further marginalize those most vulnerable and underserved in medicine. Renewed attention to the Common Rule in the wake of this update presents an opportunity to address some of the many historical and social determinants that have led to long-standing health inequities. We propose several considerations for IRBs and the research community at large that address the interests of communities and underscore the principle of justice. Our recommendations seek to amend human subjects' protections that currently overemphasize individualism and autonomy to include a collectivist ethos that is at least equally as valued in communities of color and has been critical in the historical narrative of oppressed groups. IRBs and investigators can mitigate heath inequities first by recognizing their connection with research, and second by better addressing the role and interests of underrepresented individuals and their communities in research.

We use the term "health inequities" to refer to systematic inequalities in health whereby disadvantaged social groups (e.g., the poor, racial or ethnic minorities, women, the mentally disabled, and other groups) suffer worse health and decreased access to health care compared with more advantaged social groups. ${ }^{2,3}$ The social determinants of health-that is, the circumstances in which people are born, grow, live, work, age, and die-are governed by the distribution of power, wealth, and resources, and thus are largely responsible for health inequities. Health inequities have been present throughout history. In the United States, African American sociologist W.E.B. DuBois described and elucidated racial disparities in health as early as the 19 th century. ${ }^{4}$ Throughout American history, medical experimentation has used individuals from underserved and marginalized groups as involuntary research subjects, including enslaved Africans, members of the military, women and children, prisoners, mentally disabled individuals, and other groups vulnerable because of social, economic, or medical conditions. Finally, in the aftermath of the U.S. Public Health Study at Tuskegee in 1974, the National Commission for the Protection of Human Subjects of Biomedical and Behavioral Research was created. Since the commission's issuance of the Belmont Report in 1978, those invested in the ethical conduct of research have tried to strike the balance between the need to advance medical practice and the need for ethically sound engagement of diverse groups in research. This balance requires thoughtful attention to the Belmont Report's principle of justice, which demands that disadvantaged and potentially vulnerable groups are neither inappropriately included in nor inappropriately excluded from research.

Much progress has been made. Although the Common Rule still references "subjects," terminology in research ethics more broadly has moved from "subject" to "participant" to signal the voluntary nature of research participation. Regulations now require that groups considered vulnerable or protected-minorities, women, and children-nevertheless have the opportunity to participate in and reap the potential benefits of research. In the last decade, we have seen growing interest in and momentum around patient-centered research and engaged research approaches.

Engaged research approaches including community-based participatory research, community-engaged research, and community-academic partnered participatory research were born out of a recognition that only by explicitly including marginalized groups as partners in research can health inequities be reduced. ${ }^{5-7}$ Such engaged approaches explicitly address concerns that research can and does contribute to social injustice and health inequities by unfairly targeting or excluding certain groups or by positioning certain populations to be less likely to see the benefits of research. As more community-academic research is pursued, it is becoming apparent that communities are entities that are more than the sum total of the individuals within them. The term "community" references a particular type of group, characterized by any of a wide variety of human associations such as geography, race, ethnicity, gender, illness or other health conditions, common culture and traditions, shared history, or religion. Communities can also be groups with a common interest or cause, or a common economy and shared resources. ${ }^{5,8}$ Communities may or may not have political authority (as in the case of some American Indian tribes) and will have varying degrees of cohesiveness with regard to specific characteristics that define a community. ${ }^{8}$

Communities are not explicitly protected from harm by the Common Rule. Although protections for individuals are absolutely essential for the conduct of ethical medical research, potential group harms and benefits should also be considered during both study design and study review processes. On the surface, this may seem at odds with the principle of 
autonomy, which is largely interpreted as being applicable to individuals. However, consideration of group harms and benefits expands the ethical dialogue, addresses expectations of a favorable risk-benefit ratio not only for individuals but for communities as well, and emphasizes the creation of scientific and social value in research.

The updated rule acknowledges community-level concerns and the potential for group harms and benefits only with respect to American Indian and Alaska Native populations (at $\$ 46.101$ (f) acknowledging applicability of tribal law in addition to the Common Rule). The preamble to the Final Rule notes that American Indian and Alaska Native communities have a long history of group consent, tribal consent in addition to individual consent, and even tribal research review that considers "community-level protections important for maintaining the integrity of culturally significant information and practices." Apart from this, however, the Department of Health and Human Services missed the opportunity to codify into the
Final Rule the consideration of community-level concerns as fundamental to ethical research for all communities, especially those who have been historically marginalized or underserved.

Table 1 summarizes key changes to the Common Rule as adopted in the Final Rule, and provides recommendations for further IRB guidance that would advance the principle of justice in research, recognize the shifting role and expertise of stakeholders and communities in research, and establish protections that address community concerns. These recommendations are detailed as follows:

- The most significant and useful change would be to consider "communities" as "subjects." Throughout the text of the Common Rule, wherever there is reference to "subject/s," researchers and IRBs should interpret this to mean not only individuals, but also communities. This understanding would necessarily mean that, for IRB approval, consideration of the risks and benefits to "subjects" should include group harms and benefits.

Table 1. Major Updates to the Common Rule and Community-Oriented Recommendations for IRBs and Researchers

Common Rule

$\$ 46.101$ (b)

Exempt research

$\$ 46.107$ IRB

Membership

$\$ 46.109$ IRB review of research

$\$ 46.111$ Criteria for IRB approval of research

$\$ 46.112$ Review by institution

$\$ 46.114$ Cooperative research

$\$ 46.116$ General requirements for informed consent

Updated Final Rule

Community-Oriented Recommendations

$\$ 46.104$ Adds new categories of exempt research

No significant changes

Minor language changes around the meaning of vulnerability with respect to coercion and undue influence

Eliminates continuing review for many studies

Minor changes to match other major changes

No change

Requires use of a single IRB for multisite studies in the United States, unless otherwise required by law

Adds new elements of informed consent Requires essential elements of informed consent to be presented up front Allows for broad consent for future use of stored biospecimens
Encourage/allow flexible membership of the IRB or IRB reconstitution to ensure appropriate expertise based on the type of research being reviewed.

Revise guidelines for composition, knowledge, expertise, flexibility, and training of IRBs to include communities and all forms of collaborative research.

Communities should be considered as "subjects" in addition to individuals.

"Equitable" selection of subjects should consider issues of fairness/justice as well as science. Significant discussion among academic researchers and members of affected communities may be required to agree on what constitutes fair and just.

Encourage institutions and IRBs to consider community-level concerns when approving studies.

"Cooperative research" should be interpreted to include research done in partnership with communities. Encourage/allow flexible IRB membership as above.

Include potential risks and benefits to participants' communities in consent forms.

Broaden the concept of informed consent to include a process for community consultation and, when appropriate, community consent.

Abbreviations: IRB, institutional review board. 
- The Final Rule includes a directive for use of a single IRB. This increases the relative importance of any given IRB's membership. Although the Common Rule directs that IRBs should include members "with varying backgrounds to promote complete and adequate review of research activities commonly conducted by the institution" and allows for consultation with "individuals with competence in special areas to assist in the review of issues which require expertise beyond or in addition to that available on the IRB," flexible membership - that is, allowing an IRB to reconstitute its voting members as needed-would ensure that the IRB is able to conduct an "informed review" tailored to the type of research being considered.

- "Cooperative research" should be interpreted to include research done in partnership with communities, not just multi-institution studies.

- The "equitable" selection of subjects should explicitly consider issues of justice and fairness as well as science. A study's selection process should be designed such that vulnerable or disadvantaged groups are not exploited, nor are they excluded without sufficient scientific justification to do so. Benefits and burdens should be distributed fairly, with the judgment of "fair" taking into consideration the views of the affected population(s). Equitable selection of participants from communities that experience disparities should have a fair process for joint decision making among community members and academic partners. Research teams need to be cognizant of the difficult question of what constitutes fair and just and be willing to discuss issues and work out jointly acceptable solutions. ${ }^{5}$

- All researchers should be encouraged to include a statement describing the potential group benefits and group harms in the text of informed consent forms. This measure would ensure that researchers thoughtfully consider communitylevel impacts in study design and outcomes, and would also acknowledge individuals' roles in their larger communities. Explicit acknowledgment of individuals as a part of a whole is particularly valuable for historically marginalized communities, not only for building trust but necessary for addressing health inequities.
- Informed consent can and should include a process for community consultation and, when appropriate, community consent. Community consultation and consent go hand in hand. Community consent involves approval of the research proposal by a governing body with the authority to enter into binding agreements on behalf of the community, as is the case for many American Indian and Alaska Native populations. If there is no formal governing body, community consent is not possible. In this case, additional protection can occur in the form of consultation. Community consultation involves varying levels of engagement of members of the community of focus in some or all aspects of the research process. Examples of consultation include seeking input from local leaders (formal or informal) and community members regarding the acceptability of specific aspects of the research proposal, the most effective ways to recruit participants from a given community, or how to disseminate research findings so as to reach the greatest number of people potentially affected. ${ }^{8}$

The updated Common Rule acknowledges the changing landscape of research and draws renewed broad attention to research ethics. However, it does not robustly incorporate into its directives consideration of the social determinants of health or the impact of health inequities. Now is the opportune time to encourage researchers and IRBs to interpret the new guidelines in ways that are more inclusive, consider protection of communities as well as individuals, and broaden the concept of informed consent to include group harms and benefits.

\section{ACKNOWLEDGMENTS}

The authors thank Patricia King, Kiana Bess, James Coleman, and Shristi Tiwari for their helpful input and contribution to the development of ideas reflected in this paper. The research for this paper was financially supported by grants from the Greenwall Foundation Grant and the National Institutes of Health (K24 HL105493). 


\section{REFERENCES}

1. Department of Health and Human Services. NPRM for federal policy for the protection of human subjects [updated 2015]. http://www.federalregister.gov/articles/2015/09/08/201521756/federal-policy-for-the-protection-of-human-subjects

2. Braveman P. Health disparities and health equity. Concepts and measurement. Annu Rev Public Health. 2006;27:167-94.

3. Whitehead M. The concepts and principles of equity in health. Int J Health Serv. 1992;22:429-45.

4. Williams DR, Sternthal M. Understanding racial/ethnic disparities in health: Sociological contributions. J Health Soc Behav. 2010;5(Suppl):S15-S27. http://doi.org/10.1177 /0022146510383838

5. Chen DT, Jones L, Gelberg L. Ethics of clinical research within a community-academic partnered participatory framework. Ethnicity Dis. 2006;16:118-33.
6. Cacari-Stone L, Wallerstein N, Garcia AP, Minkler M. The promise of community-based participatory research for health equity: A conceptual model for bridging evidence with policy. Am J Public Health. 2014;104(9):1615-23.

7. Wallerstein N, Duran B. Community-based participatory research contributions to intervention research: The intersection of science and practice to improve health equity. Am J Public Health. 2010;100(S1):S40-6.

8. Weijer C, Emanuel EJ. Protecting communities in biomedical research. Science. 2000;289(5482):1142-4.

9. U.S. National Archives and Records Administration. Fed Reg. 2017;82(12):7158. 